\title{
Haptic Feedback Improves Manual Excitation of a Sprung Mass
}

\author{
Felix Huang R. Brent Gillespie Art Kuo \\ Department of Mechanical Engineering, University of Michigan, Ann Arbor, MI 48109 \\ fhuang@umich.edu; brentg@umich.edu; artkuo@,umich.edu
}

\begin{abstract}
In this paper, we present an experiment in which human subjects were asked to manually excite a virtual sprung mass into resonance under various feedback conditions: visual, haptic or visual and haptic combined. We are interested in comparing the value of these feedback conditions in terms of their influence on the achievable performance in a dynamic task such as exciting a resonant mechanical system. From our human subject experiment $(n=10)$, we found that with haptic feedback alone, subjects successfully excited the sprung mass into resonance. For the particular case of $\omega_{\mathrm{n}}=7 \mathrm{rad} / \mathrm{s}$, subjects demonstrated significantly larger differences between the observed and expected frequency distribution under vision-only (paired t-test: $p=0.034$ ) and haptics-only feedback conditions (paired t-test: $p=0.021$ ), as compared to combined vision with haptic feedback. Variability of key marker locations of input behavior were also significantly lower with both feedback channels than with either alone (paired t-tests: $p<.0002)$. Our results show that haptic feedback can augment vision to produce significant improvements in the control of a dynamic system.
\end{abstract}

\section{Introduction}

Certain percepts are better served by some sensory modalities than others. For example, color can be seen but not felt and pitch can be heard but not seen. Also, texture, while often visible, is usually more easily recognized by touch than by vision. A natural question to arise among researchers in haptics is: "What percepts are best served by the haptic senses?" We believe that some of the most inherently haptic of percepts are those associated with the identification of properties of dynamical systems-- properties such as inertia, natural frequency or damping ratio, for example. A dynamical system may be defined broadly as any system with memory, or a system whose model includes a state that encodes the effect of past inputs. Thus the response of a dynamical system depends not only on the present input, but also on the past input. We believe that the haptic sensory and perceptual system is particularly well suited to handle the interpretation of signals whose invariants lie in both time and space. Further, in that haptics involves both excitation and response of mechanical systems, it seems that all elements are present in the human haptic apparatus to form a particularly effective tool for dynamical system identification.

Dynamical systems containing free motion or at least one degree of freedom distal to the point of grasp are under-actuated-- to borrow a term from the field of robotics (Lynch, Mason[1, 1996)]. This distal degree of freedom makes manual control of under-actuated systems more difficult than a reaching or aiming task. Rather than simply making a discrete motion to position the hand (Meyer[2] at al., 1999), one must act through the system dynamics to position the part of the dynamical system that is not directly grasped. Such a task might be called an under-actuated task. Control action from the arm may be sufficient to cause the appropriate evolution of the un-actuated degrees of freedom given the proper controller strategy.

However, because humans cannot use proprioception to sense all states of the systems to be controlled, other afferents such as visual or haptic feedback become necessary. Our goal in this study is to show how haptic feedback may serve as the sole feedback channel as well as a channel that augments vision in a continuous, under-actuated task. We explore the prototype task of manual excitation of a virtual sprung mass.

Haptic feedback may certainly be important in cases where vision is limited, acting as a supplementary feedback channel. For many upper extremity tasks, however, vision alone is sufficiently rich to meet task goals (Todorov, R. Shadmehr, \& E. Bizzi[3, 1997)]. Ernst, on the other hand, has shown that humans can combine haptic and visual information for improved psychophysical perceptivity of texture properties (Ernst et al.[4, 2002)]. For the purposes of perception, haptic feedback can readily provide more information that may be useful to the human. As opposed to using psychophysical surveying, the current study explores the effects of haptic and visual integration on measurable task performance in the control of an under-actuated dynamic system.

We are interested in studying the effects of haptic feedback on manual tasks with continuous dynamics and force interactions. In previous work, 
we studied human operator control of a ball and beam system and found that skill transfer to a target system with a real ball was improved if training on a virtual system included similar force interactions as those of the real system (Huang, Kuo, Gillespie[5, 2002)]. We found, however, that the ball and beam task was visually dominated and was difficult if not impossible to perform without vision. Rhythmic bouncing has been studied by Schaal et al. showing superior performance with the use of haptic feedback alone as compared with vision alone (Schaal et al.[6, 1996)]. These results, however, cannot be generalizedare difficult to generalize-one reason being that the scaling of visual and haptic feedback intensity will depend on the particular task. In addition, the information and power transfer characteristics of discrete impacts are very different than those of continuous tasks. In this study we will begin to form a generalized understanding of the role of manual performance in a simple continuous task where the relative scaling of haptic and visual feedback are controlled.

Though haptic feedback can carry information useful for control, it might also come at a cost. When haptic feedback is present, there exists mechanical coupling: a feedback loop is closed involving force and motion. Not just information, but also power may then be transferred across the mechanical contact. The amount of mechanical work expended by the human due to haptic feedback, if considered a cost, must be compared to the profit associated with acquisition of information that becomes available with the addition of haptic feedback. However, if the human arm cannot cope with the force interactions during continuous contact, unintended motion might occur.

As an example of how humans cope with and even make use of interaction forces, past research has shown that humans can exploit the spring like behavior of muscles for useful behavior by modulating the effective stiffness or by changing the equilibrium orientation of limbs. Burdet has shown that humans can modify the effective impedance of the arm when practicing aiming movements in a destabilizing force field (Burdet[7, 2001)]. Mah studied reaching tasks with randomly presented virtual walls, and found results suggesting changes in the effective joint stiffnesses of the upper extremity during movement (Mah[8, 2001)]. Haptic feedback would, therefore, have an advantage over vision, since using modulation of effective joint impedances as strategy for motor control would not be useful unless force interactions were in fact present.

For an unknown dynamical system, identification of system parameters derived from haptic or visual information could be used to serve an internal system model (Kawato[9, 1999)] in the mind of the human operator. Kuo developed a model of rhythmic limb movements employing feedforward and feedback elements that exhibits robustness against disturbances $(\mathrm{Kuo}[10,2002)]$. A control strategy that is tailored to the specific underactuated system may be used to plan forces for mechanical efficiency. Dingwell studied human control of a sprung mass with haptic feedback, where the goal was to position the mass in a target box (Dingwell[11, et al., 2002)]. He found that through the use of "catch trials", that humans use strategies consistent with the use of a lowimpedance controller and internal model. In the manual excitation of a sprung mass, human operators may use a simple parameterization of the system dynamics, such as identifying the resonant frequency or the size of the sprung mass.

Our approach in the current study is to show the contributions of visual and haptic feedback on quantitative performance metrics. In the current study, we ask normal, healthy human subjects to manually excite a sprung mass, a simple example of an under-actuated system. We chose resonant excitation as the control goal for the human subjects. Successful identification of this system requires operating at the correct natural frequency. Visual or haptic feedback could both be potentially used to provide the operator with information about the system. Feedback of the position, velocity, or even the phasing of the sprung mass relative to the input handle could be used for the purposes of error correction or identification. Without discriminating between the use of feedback for identification or for error-correction, we will show that haptic feedback alone is sufficient to perform the resonance task proficiently, and that haptic feedback can be used to augment vision and improve overall performance. In the following sections we present an analysis of the sprung mass system equations of motion, the implementation of the virtual environment, and the design of the experimental protocols. We then develop our performance metrics used to gauge the success of our human subjects in the manual excitation of the sprung mass. Finally, we present the sample results and a discussion of our human subject study.

\section{Methods}




\subsection{Sprung Mass Equations of Motion}

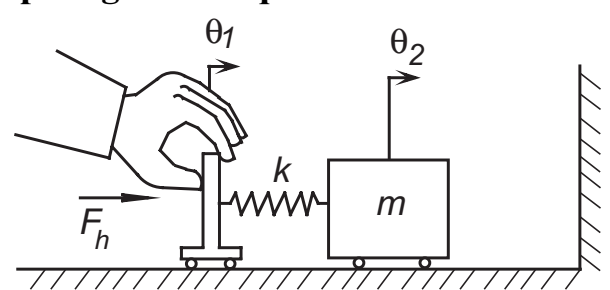

Figure 1. Schematic of sprung mass and lightweight handle

In this section we present the virtual environment used in our human subject study. Consider the linear system in Figure 1 consisting of a sprung mass whose displacement $\theta_{2}(\mathrm{t})$ is driven by the displacement $\theta_{1}(\mathrm{t})$ of a handle which is driven in turn by the user's hand. We use $m$ and $k$ as the parameters of the sprung mass and consider the handle massless. The equation of motion and output equation are:

$$
\begin{gathered}
\ddot{\theta}_{2}(t)+\omega_{n}{ }^{2}\left(\theta_{2}(t)-\theta_{1}(t)\right)=0 \\
F_{h}(t)=-k\left(\theta_{2}(t)-\theta_{1}(t)\right)
\end{gathered}
$$

where $\omega_{n}^{2}=k / m$, and $\mathrm{F}_{\mathrm{h}}$ is the interaction force between the arm and the handle. Using Euler's method to discretize this model, we produced a virtual representation of the sprung mass attached to the handle of our haptic interface. Since in fact our interface has a rotary handle, we used the rotational analog of the system in figure 1 and displayed on the computer screen two beams that rotated about their centers on a common fixed pivot. The handle was animated as a small blue beam while the rotary analog of the sprung mass was a large green beam. The spring connecting the beams was not displayed. In order to provide a challenging visual task, yet provide feedback faithful to the dynamics, the separation of the sprung mass and handle was reduced by a scale factor. We set the scaling to 0.0075 of the simulated $\theta_{2}(t)$ response. This scaling avoided angular wrapping of the sprung mass and thus making made the animation much easier to read, but reducing reduced the overall visual resolution. Based on subject performance in pilot experiments, we attempted to balance the contribution of visual feedback relative to haptic feedback. The angular displacement of the handle was not rescaled.

For the virtual sprung mass, energy injected by the user can be stored as either potential energy in the spring or as kinetic energy in the mass. Stored energy is returned to the user through the handle or dissipated in the physical damping of the haptic interface. Equation 1 shows that the acceleration of the output mass is proportional to the spring extension. From Equation 2, with the assumption of a massless interface handle, the force felt by the operator at the handle is equal in magnitude to the spring force. For various settings of mass and spring stiffness, we can obtain responses that are kinematically equivalent (same $\omega_{n}$ ) but have different gains on haptic feedback. That is, the haptic feedback magnitude may be set using the spring constant. For a given value of the spring stiffness $k$, we set the system natural frequency using the value of $m$.
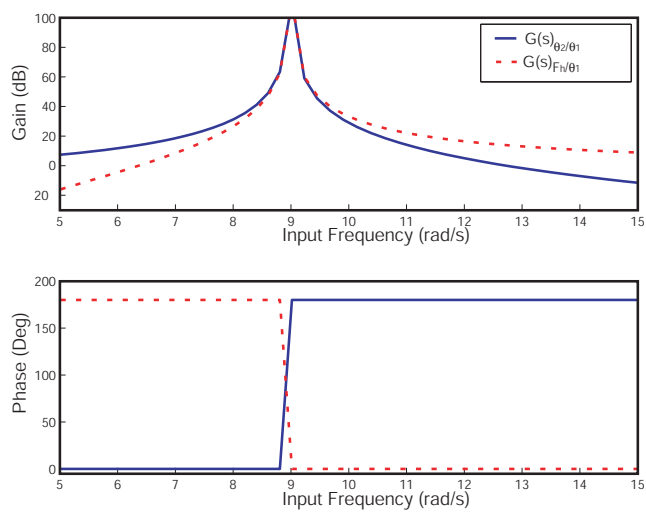

Figure 2. This sample Bode plot of the sprung mass $\left(\omega_{n}=9\right.$ $\mathrm{rad} / \mathrm{s}$ ) shows that the force interaction $F_{h}$ and the mass position responses $\theta_{2}$ to handle input motion $\theta_{1}$ achieve highest gain at resonant frequency $\omega_{\mathrm{n}}$.

The sprung mass has simple dynamics. Assuming that the operator applies a constant amplitude sinusoidal motion to the handle, the sprung mass motion and interaction force are expected to achieve high gain near the resonant frequency in steady state (see Figure 2). The haptic feedback due to this force at the handle and the visual feedback of the sprung mass both should provide information to the user about the resonant frequency of the sample sprung mass. When the user drives at the natural frequency, the phase of both feedback channels will lag the input handle position by 90 degrees in steady-state response.

\subsection{Description of Apparatus and Implementation of Virtual Environment}




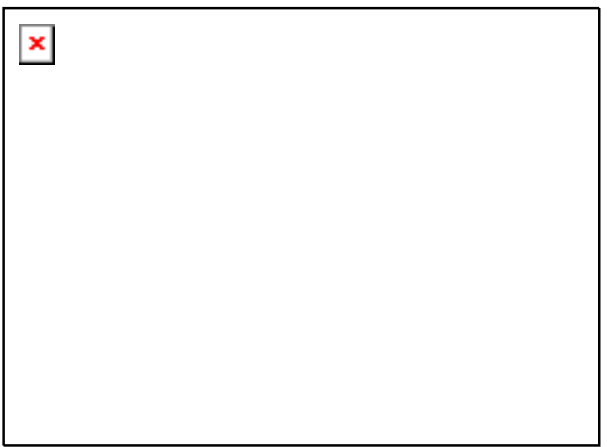

Figure 3. Operation of virtual sprung mass resonance task may include visual and haptic feedback.

We designed and constructed a single degree of freedom manual interface with a rotating handle that a human operator can grasp with the hand and turn with pronation/supination movements of the forearm. The T-shaped handle is mounted on a gear, which is driven by a DC-motor through a chain and sprocket assembly. An optical encoder is used to sense the angular position of the handle with a resolution of 2048 counts per revolution. The device is housed in a protective acrylic casing that allows portable desktop usage and adjustments for posture. A desktop PC collects the measured data and controls the motor in real-time with a sampling rate of $1 \mathrm{kHz}$. Experimental data are logged at a rate of $100 \mathrm{~Hz}$.

Our experimental setup allows visual and haptic feedback to be turned on or off according to the experimental protocol. For the current study the spring constant is fixed $(\mathrm{k}=0.0125 \mathrm{~N}-\mathrm{m} / \mathrm{rad})$, to keep the haptic feedback scaling constant. The natural frequencies presented were varied by changing the value of the virtual mass.

\subsection{Design of Experimental Protocol}

\begin{tabular}{|c|c|}
\hline $\begin{array}{l}\text { Number of } \\
\text { Subjects }\end{array}$ & $\mathrm{n}=10$ \\
\hline $\begin{array}{l}\text { Feedback } \\
\text { Conditions: }\end{array}$ & $\begin{array}{ccc}\text { Vision-Only, } & \text { Haptic-Only, Vision-Haptic } \\
(\mathrm{VX}) & (\mathrm{XH}) & (\mathrm{VH}) \\
\end{array}$ \\
\hline $\begin{array}{l}\text { Undamped } \\
\text { Natural } \\
\text { Frequencies }\end{array}$ & $\begin{array}{c}7,9,11, \text { and } 13 \mathrm{rad} / \mathrm{s} \\
(1.11, \quad 1.43, \quad 1.75, \text { and } 2.06 \mathrm{~Hz})\end{array}$ \\
\hline $\begin{array}{l}\text { Spring } \\
\text { Stiffness: }\end{array}$ & $0.0125 \mathrm{~N}-\mathrm{m} / \mathrm{rad}$ (visual angle). \\
\hline Trial Duration: & 30 seconds \\
\hline $\begin{array}{l}\text { Number of } \\
\text { Trials }\end{array}$ & 3 replicates, 12 conditions for 36 trials \\
\hline
\end{tabular}

Our experiment design compared the success in operating the virtual sprung mass at the resonant frequency for three feedback conditions:
Vision (VX), Haptic (XH), and Vision-Haptic (VH). In trialsthe $\mathrm{XH}$ trials, visual feedback of only the handle position was included. In VH and VX trials, visual feedback of the handle position and the mass position was provided. The feedback condition and the system natural frequencies (See Table 1) were presented in randomized trials for each subject. Ten normal, healthy adults ( 7 male, 3 female) participated in this study after providing informed consent.

The goal of the manual task was to find the system's natural frequency as quickly as possible and operate the handle at that frequency smoothly. Subjects performed the task while seated and were given instructions on arm and hand posture. Using the dominant hand, subjects grasped a motorized handle with elbow resting on a padded table top, and operated the handle using arm pronation and supination (See Figure 1). Subjects were also instructed to attempt to keep the handle motion bounded within 30 degrees of the vertical as shown by markers in the animation.

For each trial, color changes in the animation and beeps signaled to the operator that the computer program was changing between the following modes: a) trial ready (white) b) trial running (green), c) trial stop (red). Rotating the handle counter-clockwise toward the horizontal unlocked the program so that then the next trial run was ready (a). As the operator turned the handle toward the vertical, the sprung mass stretched to a deflection of 45 degrees and is was released. Upon release, the trial began and data was collected (b). After 30 seconds the trial ended and the sprung mass position became locked to the handle (c). The next trial was then unlocked in the same manner as before. For this experiment the subject was allowed to begin the next trial at any time, so that the resting period between trials was not controlled.

\subsection{Development of Performance Metrics}




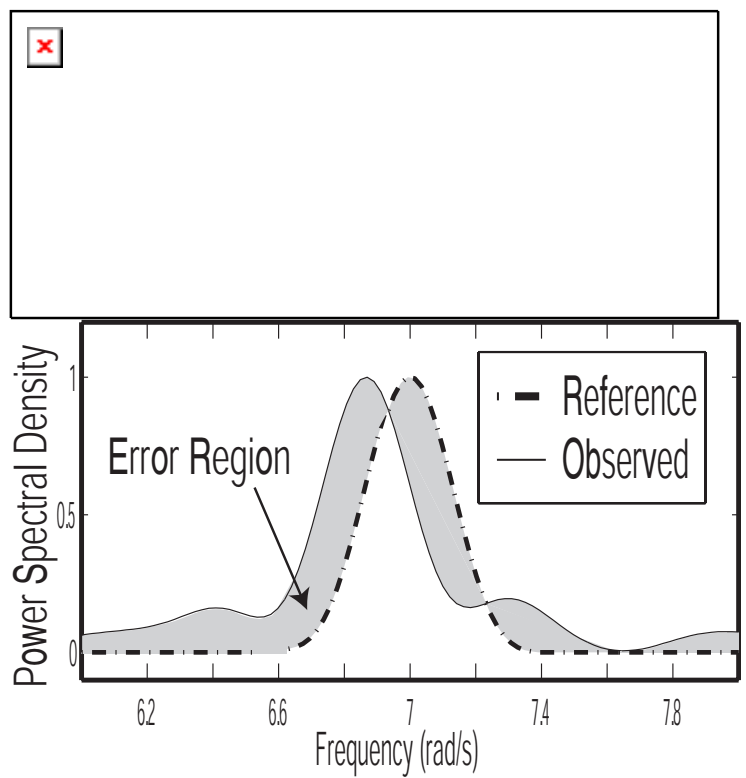

Figure 4. These frequency distributions of handle input motion show an error between the observed and a reference spectrum at a target $7 \mathrm{rad} / \mathrm{s}$. Sample data from a single subject is shown.

As a performance metric, we calculated the sum of squared differences (SSD) between the observed and reference power spectral density distributions (See Figure 2) of the sprung mass displacement. The SSD is a measure of how the frequency content of the sampled handle motion compares to that of an ideal sampled sinusoid.

$$
S S D=\sum_{i=1}^{N / 2}\left(P_{\theta_{1}}\left(j \omega_{i}\right)-P_{r e f}\left(j \omega_{i}\right)\right)^{2} \quad \text { Eqn. } 3
$$

The reference distributions, denoted by $\mathrm{P}_{\text {ref, }}$ were determined from pure sinusoids, simulating handle input position data for each target natural frequency (100 Hz sample rate). Using collected handle position data from each trial, we determined the power spectrum, denoted by $\mathrm{P}_{\theta 1}$, of the handle position (also sampled at $100 \mathrm{~Hz}$ ). From the 30 seconds of the simulated or experimental trial data ( $\mathrm{N}=3000$ data points), we produced estimates of the power spectral density using an averaged periodogram method (see 'pwelch' function MATLAB Signal Processing Toolbox). We used an $\mathrm{N}$ point Hamming window and $2^{14}$ point FFT. Using these settings, the SSD metric showed a linear relationship to the differences in frequency between two ideal sampled sinusoids within a region of + $1.5 \mathrm{rad} / \mathrm{s}$ about the target natural frequency.
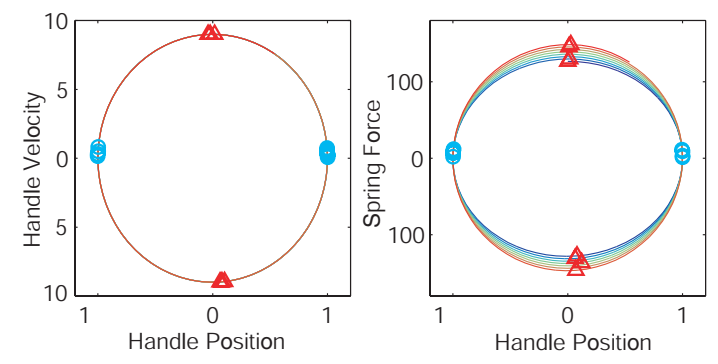

Figure 5. Simulated response plots of the sprung mass system (10 seconds with initial stretch of $\mathrm{X0}=50$ ) show expected marker locations for each occurrence of zzero sprung mass velocity (triangles) and zero spring force (circles) as would be expected by the steady state phase relationship between sinusoidal input motion and the sprung mass output motion .

A second performance metric is available in the qualitative and quantitative properties of the handle position-velocity plot. Assuming ideal sinusoidal input from the handle, we expect key features to appear in the steady state, such as a 90 degree phase relationship between the position of the handle and the sprung mass. As simulations of the sprung mass system show, input sinusoidal motion driven at the natural frequency (see Figure 5) causes the sprung mass displacement maxima (or zeros of sprung mass velocity) to occur close to handle position zero-crossings. Similarly, occurrences of zero spring force take place near the occurrence of zero handle velocity. In addition to the marker locations, a smooth elliptical shape of position-velocity plot is predicted if the handle position is perfectly sinusoidal. To analyze the variability of human subject performance in the sprung mass excitation task, we calculated the standard deviationsvariability of the marker locations of for zero sprung mass velocity and zero spring force in theas they occur in the handle position-velocity plots.

\section{Results}

\subsection{Sum Squared Differences Metric for all Natural Frequencies}

We found that the sum-squared differences metric demonstrated some cases of significantly better performance when haptic feedback was included. See Figure 3 for mean results of frequency distribution sum-squared differences for each condition. Vision-Only (VX) trials produced significantly more error than Haptic-Only $(\mathrm{XH})$ trials for frequency conditions 7, 9, $13 \mathrm{rad} / \mathrm{s}$ (paired t-test, $\mathrm{p}<0.005$ ). Vision-Only trials produced 
significantly more error than Vision-Haptic (VH) trials for all frequencies tested (paired t-test, $\mathrm{p}<$ 0.002 ). VisionHaptic-Only trials produced significantly more error than Vision-Haptic trials in the $\omega_{\mathrm{n}}=7 \mathrm{rad} / \mathrm{s}$ case (paired t-test, $\mathrm{p}=0.021$ ). Haptics-Only trials produced significantly more error than Vision-Haptic (VH) trials for all frequencies tested (paired t-test, $\mathrm{p}<0.002$ ). For frequencies other than $7 \mathrm{rad} / \mathrm{s}$, the combined feedback condition Haptic (VH) was not found to be significantly different than the haptic alone condition Haptic $(\mathrm{XH})$.

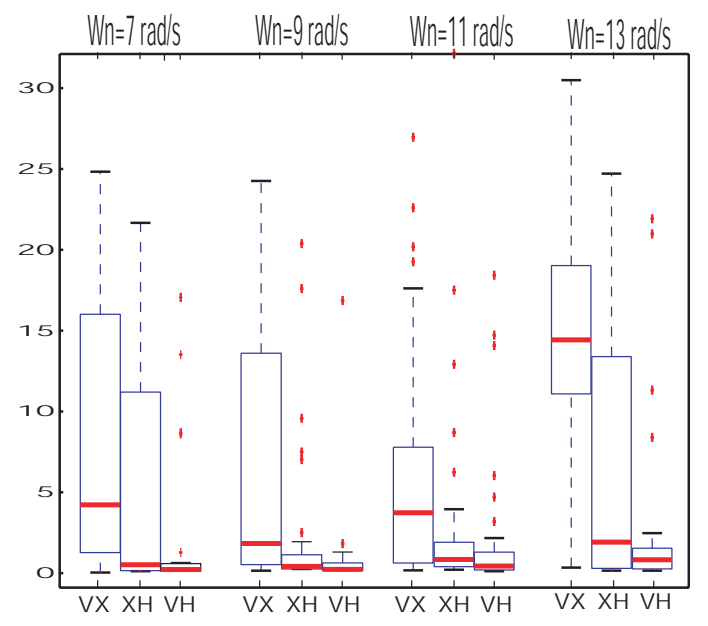

Figure 6. The group mean SSD results show an overall trend of better performance and lower group variance with haptic feedback and with combined feedback. The box and whisker plots indicate the median, first and last quartiles of the group mean SSD values.

\begin{tabular}{|l|llll|}
\hline \multicolumn{5}{|c|}{$\begin{array}{c}\text { Summary of paired Student's t-test } \mathbf{p} \text {-values } \\
\text { comparing SSD between feedback conditions }\end{array}$} \\
\hline & \multicolumn{1}{|c|}{$7 \mathrm{rad} / \mathrm{s}$} & \multicolumn{1}{c|}{$9 \mathrm{rad} / \mathrm{s}$} & $11 \mathrm{rad} / \mathrm{s}$ & $13 \mathrm{rad} / \mathrm{s}$ \\
\cline { 2 - 5 } $\mathrm{VX}>\mathrm{VH}$ & $\mathbf{0 . 0 0 0 1 1}$ & $\mathbf{0 . 0 0 3 7}$ & $\mathbf{0 . 0 0 1 3}$ & $\mathbf{0 . 0 0 0 1 8}$ \\
$\mathrm{XH}>\mathrm{VH}$ & $\mathbf{0 . 0 2 1}$ & 0.19 & 0.5 & 0.16 \\
$\mathrm{VX}>\mathrm{XH}$ & $\mathbf{0 . 0 3 4}$ & $\mathbf{0 . 0 4 1}$ & 0.081 & $\mathbf{0 . 0 0 0 3 7}$ \\
\hline
\end{tabular}

Table 21. The SSD metric showed significantly better performance $(p<.05)$ with the inclusion of haptic feedback and for combined feedback at the $\omega_{\mathrm{n}}=7 \mathrm{rad} / \mathrm{s}$ condition.

\subsection{Sample Trajectories and Marker Variability}

We next present an analysis of the variability of the handle motion as a function of the feedback condition. A sample of the positionvelocity and the position-force plot for one subject (See Figure 7) demonstrates typical differences by condition in the shape and in the overall variability of the trajectories. Qualitatively, there appears to be more convergence of the cycles in the conditions where haptic feedback is present and when both feedback channels are available. Some subjects, however, demonstrate a characteristic warping of the position-velocity plot when haptic feedback is present that occur as indentations in the traces in the first and third quadrants. For these cases, the marker positions of the position-velocity plots indicating points of zero sprung mass velocity and zero spring force show deviation from the expected positions that were discussed above in the methods section.

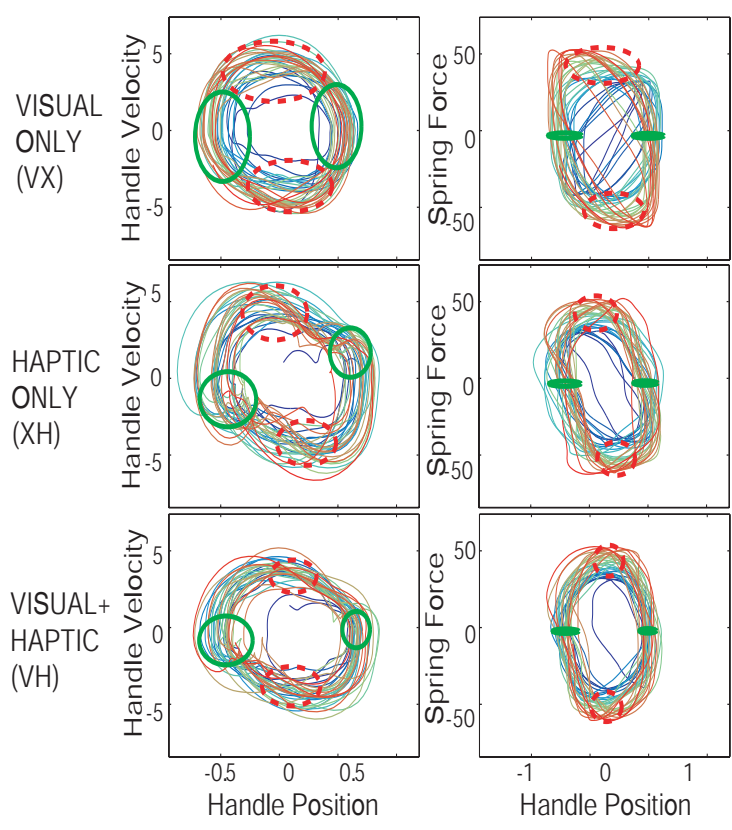

Figure 7. These sample plots (for 1 subject, three separate 30 second trials) of handle position versus velocity (left) and handle position versus spring force (right) show typical differences in the trajectory shape and data variance due to feedback conditions. The handle position and velocity variability at zero sprung mass velocity (dashed) and zero spring force are shown with ellipses radii of 3 standard deviations.

We found significant differences in the marker position variability as a function of the feedback condition. As representative metrics, we compare the group mean standard deviations of the handle position at zero sprung mass velocity ( $\left.\hat{\theta}_{1} @ \dot{\theta}_{2}=0\right)$ as well as that of the handle position at zero force $\left(\hat{\theta}_{1} @ \dot{F}=0\right)$ (See Figure 8). A Student's ttest (See Table 2) confirms that the significantly lower variability for these markers was achieved for combined feedback compared to vision alone for nearly all frequencies tested $(\mathrm{p}<.03)$. The combined 
feedback case also resulted in significantly lower variability than with haptic feedback alone for all frequencies tested $(p<0.002)$. The haptic only condition also resulted in better consistency than with vision alone in many cases.

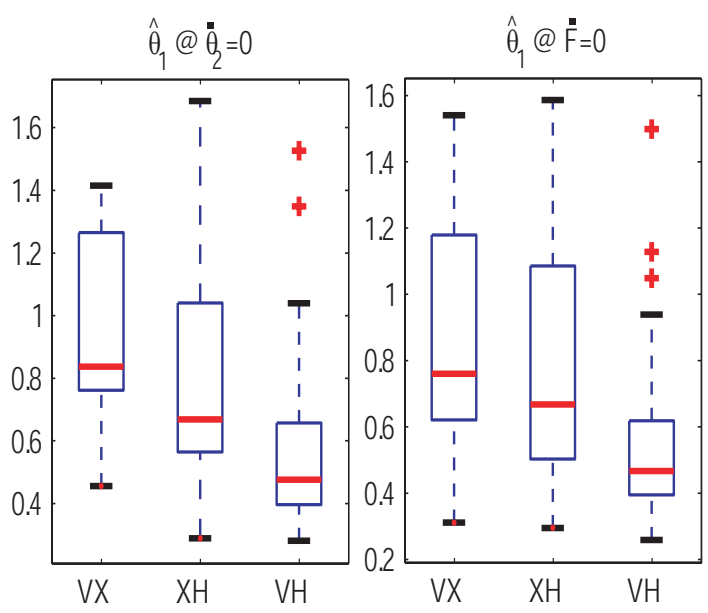

Figure 58. The box and whisker plots indicate the median, first and last quartiles of the group mean standard deviations of marker positions $\left(\omega_{n}=7 \mathrm{rad} / \mathrm{s}\right.$ condition $)$. The handle positions at zero sprung mass velocity and zero spring force show an overall trend of lower variance with haptic feedback and with combined feedback.

\begin{tabular}{|c|c|c|c|}
\hline \multicolumn{4}{|c|}{$\begin{array}{c}\text { Paired Student's ttest p-values } \\
\text { comparing marker standard deviations } \\
\text { between feedback conditions }\end{array}$} \\
\hline \multirow{2}{*}{$\omega_{\mathrm{n}}$} & Test & $\hat{\theta}_{1} @ \dot{\theta}_{2}=0$ & $\hat{\theta}_{1} @ \dot{F}=0$ \\
\hline \multirow{2}{*}{$7 \mathrm{rad} / \mathrm{s}$} & $\mathrm{VX}>\mathrm{VH}$ & $<0.001$ & $<0.001$ \\
& $\mathrm{XH}>\mathrm{VH}$ & 0.013 & 0.006 \\
& $\mathrm{VX}>\mathrm{XH}$ & 0.026 & 0.246 \\
\hline \multicolumn{4}{|c|}{} \\
\hline \multirow{3}{*}{$9 \mathrm{rad} / \mathrm{s}$} & $\mathrm{VX}>\mathrm{VH}$ & $<0.001$ & $<0.001$ \\
& $\mathrm{XH}>\mathrm{VH}$ & 0.028 & 0.017 \\
& $\mathrm{VX}>\mathrm{XH}$ & 0.002 & 0.005 \\
\hline \multicolumn{4}{|c|}{} \\
\hline \multirow{2}{*}{$11 \mathrm{rad} / \mathrm{s}$} & $\mathrm{VX}>\mathrm{VH}$ & 0.001 & $<0.001$ \\
& $\mathrm{XH}>\mathrm{VH}$ & 0.010 & 0.067 \\
$\mathrm{VX}>\mathrm{XH}$ & 0.240 & 0.145 \\
\hline \multicolumn{4}{|c|}{} \\
\hline \multirow{2}{*}{$13 \mathrm{rad} / \mathrm{s}$} & $\mathrm{VX}>\mathrm{VH}$ & 0.002 & 0.001 \\
& $\mathrm{XH}>\mathrm{VH}$ & 0.003 & 0.010 \\
$\mathrm{VX}>\mathrm{XH}$ & 0.860 & 0.205 \\
\hline
\end{tabular}

Table 2. Group mean standard deviations of marker positions were significantly lower $(p<.03)$ for combined feedback compared to vision alone.

\section{Discussion}

The sum-squared differences (SSD) metric shows that haptic feedback acting alone provided sufficient information to allow human subjects to detect and operate the sprung mass systems at the appropriate resonant frequencies. For three of the natural frequencies tested, the haptics only condition resulted in significantly better performance than with vision only $(\mathrm{p}<.05$ for $7,9,13 \mathrm{rad} / \mathrm{s})$. Note that success in the haptic only condition implies operating at the appropriate driving frequency while generating the necessary hand forces.

For the case of $\omega_{n}=7 \mathrm{rad} / \mathrm{s}$, the combined feedback condition resulted in significantly better group mean performance in the SSD metric than with vision alone $(\mathrm{p}=.021)$ or from haptics alone $(\mathrm{p}=1.13 \mathrm{e}-5)$. Vision contributed to significant gains in performance, suggesting that both feedback channels aided in control. When vision was included compared to haptic feedback alone, the better performance can be attributed to lower uncertainty in the states of the sprung mass. This would have allowed the human operators to identify the natural frequency of the system faster or to perform corrective action to errors in phasing in a more timely fashion. The better performance of the combined feedback condition compared to haptics alone similarly could be due to lower uncertainty, though there may have been effects other than improved information because of the forces coupling the human arm and the handle. For the higher natural frequencies presented however, haptic feedback did not significantly augment vision. It is likely that human operators found the higher frequencies in general too difficult so that the feedback quality for both channels was poor.

The comparisons of the group mean variability of marker locations also show that the inclusion of haptic feedback produces more consistency in performance. For nearly all frequencies tested, the combined feedback condition resulted in lower variability of the marker locations than with vision alone $\left(\mathrm{p}<.02, \omega_{\mathrm{n}}=7,9,13 \mathrm{rad} / \mathrm{s}\right)$. Despite the decrease in handle motion variability, the presence of haptic feedback caused characteristic distortions in the position-velocity plots showing how mechanical coupling can interfere with smooth input motion. It is not clear whether the indentations in the first and third quadrants of the position-velocity plots are intentional or not. It is possible that human operators make use of these distortions in order to obtain more distinct haptic events for state estimation or timing feedback purposesinformation. These artifacts appear to decrease with the combined feedback case suggesting that such a strategy may no longer be necessary. We found in pilot experiments that careful tuning of the haptic feedback scaling was required to achieve feedback that was sufficiently information-rich yet not fatiguing to the operator. Subjects reported that force feedback levels were 
high enough so that prolonged operation with haptic feedback would become tiring. On the other hand, some subjects reported that the inclusion of haptic feedback required less attention than with vision alone. It is possible that the use of haptic feedback reduced the required mental effort, which would be a result not explicitly captured by our performance metrics.

\section{Conclusions}

Our results show that haptic feedback can serve as the solitary feedback channel in resonance excitation of a sprung mass, an example of a continuous dynamic task. Also, the current study shows that haptic feedback can be combined with vision in order to enhance overall performance. With combined feedback, human subjects used input frequencies closer to the nominal target values and with lower variability. Qualitative analysis of the human subject input motion shows, however, that despite these gains in performance, some distortions in the input handle motion may have occurred due to the mechanical coupling between the handle and the arm.

The lower variability in the handle position is evidence of a control strategy that makes use of the identified parameters of the system through consistent open loop action. It is possible that haptic feedback decreased variability because mechanical coupling facilitated the use of an impedance modulation strategy of the arm. Such a strategy would be less prone to motor noise than actively commanding changes in muscle enervation through time. In addition to identifying the appropriate driving frequency in the control of the sprung mass, humans may determine the spring stiffness or size of the mass, so that an efficient muscle activation strategy can be employed. Whether humans actively control force through muscle action or more simply modulate the effective stiffness of the arm is a question to be further explored. In future work, we also hope to compares the performance resulting from multiple settings for visual and haptic feedback scaling.

\section{References}

[1] K. M. Lynch \& M. T. Mason, "Dynamic uUnderactuated nNonprehensile mManipulation " 1996 IEEE/RSJ International Conference on Intelligent Robots and Systems, pppages. 889896, Osaka, Japan, November 1996.
[2] Meyer, D. E., Smith, J. E. K., Kornblum, S., Abrams, R. A., \& Wright, C. E. (1990) "Speed and aAccuracy Ttrade-offs in Aaimed mMovements: tTowards a Ttheory of rRapid vVoluntary aAction" In M. Jeannerod (Ed.), Attention and Performance XIII, (pp.pages 173226). Hillsdale, N.J.: Erlbaum.

[3] E. Todorov, R. Shadmehr, \& E. Bizzi, "Augmented fFeedback pPresented in a vVirtual Eenvironment Aaccelerates 1Learning in a dDifficult mMotor tTask" Journal of Motor Behaviour, 1997,Vol. 29, No. 2, 147-158.

[4] Ernst, M.O. \& Banks, M.S. \& Landy, M.S. (2002) "Humans integrate visual and haptic information in a statistically optimal way." Nature, 415, pages 429-433.

[5] Felix Huang, R. Brent Gillespie, Art Kuo, "Haptic feedback and human performance in a dynamic task" Symposium on Haptic Interfaces for Virtual Environment Teleoperator Systems 2002: pages 24-31.

[6] Schaal S., Sternard D., Atkeson C. G., "Onehanded Juggling: A dynamical Approach to rhythmic Movement Task", Journal of Motor Behavior, 1996 28,.2, pp.pages 165-183.

[7] E. Burdet, R. Osu, D.W. Franklin, T.E. Milner, M. Kawato, "The central Nervous System Stabilizes Unstable Dynamics by Learning Optimal Impedance" Nature, VOlVol. 414, 22 November, 2001, pages 446-449.

[8] Christopher D. Mah, "Spatial and Temporal Modulation of joint Stiffnesses during multijoint movement" Exp Brain Res, (2001) 125: pages 492-506.

[9] Kawato M, "Internal models for motor control and trajectory planning" Current Opinion in Neurobiology, 9, pages 718-727 (1999)

[10] Arthur D. Kuo "The relative roles of feedforward and feedback in the control of rhythmic movements." Motor Control, 2002, 6, pages 129-145.

[11] Jonathan B. Dingwell, Christopher D. Mah, and Ferdinando A. Mussa-Ivaldi, "Manipulating objects with internal degrees of freedom: 
Evidence for Model-Based Control.” J

Neurophysiol 88: pages 222-235, July 2002.

\section{Acknowledgements}

The authors thank the anonymous reviewers for their insightful comments. This work was supported in part by the Midwest Regional Rehabilitation Network (R24). 